IP Periodica Polytechnica

Transportation Engineering

43(4), pp. 199-205, 2015

DOI: 10.3311/PPtr.8093

Creative Commons Attribution (1)

RESEARCH ARTICLE

\section{Energy- and Aerodynamic Examination of Slightly Backward Leaning Impeller Blading of Small Centrifugal Compressors}

\author{
Endre Pásztor ${ }^{1}$, Béla Varga ${ }^{2 *}$
}

Received 23 March 2015; accepted 19 June 2015

\begin{abstract}
Last decade, turbochargers with maximum 50-60 mm diameter, are more and more frequently designed with slightly backward leaning impeller blading. These kind of impeller blading, comparing to the radial blading, produces higher stress and assuming the same compressor pressure ratio it needs higher tangential speed due to the impeller exit flow slip (hereafter slip). These two disadvantages are surely compensated by some kind of thermal or aerodynamic advantages. By the authors' examination, using backward leaning impeller blading, the disadvantages are compensated by the small, but the definite increase of compressor efficiency and the positive effect on compressor characteristics.

This paper, examining and comparing the above-mentioned advantages and disadvantages, tries to clear the reasons of this design trend and hopefully contributes to the further improvement of these compressors.
\end{abstract}

\section{Keywords}

Centrifugal compressor, backward leaning impeller blading, extra stress, increase of the necessary tangential speed, decrease of the compressor pressure ratio, real and virtual impeller exit flow slip, decrease of the impeller blade diffuser angle, improved efficiency, positive effect on compressor characteristics

\footnotetext{
${ }^{1}$ Department of Aeronautics, Naval Architecture and Railway Vehicles, Faculty of Transportation Engineering and Vehicle Engineering, Budapest University of Technology and Economics

${ }^{2}$ Department of Military Aviation, National University of Public Service,

Ludovika square 2., H-1083 Budapest, Hungary

*Corresponding author, e-mail: varga.bela@uni.nke.hu
}

Abbreviations

BLIB Backward Leaning Impeller Blading

CPR Compressor Pressure Ratio.

\section{List of symbols}

Symbol Unit

$\lambda \quad-\quad$ Slip factor: $\lambda=c_{2 u l} / u_{2}$

$z \quad-\quad$ Number of blades

$\beta_{2 s h} \quad$ deg Angle between the direction of relative velocity at the impeller exit and the radial direction

$c_{2 u} \quad \mathrm{~m} / \mathrm{s} \quad$ Tangential component of absolute velocity at the impeller exit

$\begin{array}{lll}u_{2} & \mathrm{~m} / \mathrm{s} & \text { Tangential speed of the impeller tip }\end{array}$

$w_{\text {ceff }} \quad \mathrm{J} / \mathrm{kg} \quad$ Effective specific work given to the compressor

$\lambda_{v} \quad-\quad-\quad$ Virtual slip factor from the backward leaning: $\lambda_{\mathrm{v}}=c_{2 u v} / u_{2}$

$T_{\text {2re }} \quad \mathrm{K} \quad$ Final temperature of the real compression process

$T_{0} \quad \mathrm{~K} \quad$ Initial temperature of the real compression process

$c_{\text {pair }} \quad \mathrm{J} / \mathrm{kgK} \quad$ Isobaric specific heat

$\begin{array}{llll}\lambda_{\Sigma} & - & \text { Total slip factor }\end{array}$

$\eta_{i s c} \quad-\quad$ Isentropic compressor efficiency

$\eta_{\text {issot }} \quad-\quad$ Isentropic efficiency of the impeller

$\eta_{\text {isgu }} \quad-\quad$ Isentropic efficiency of the stator vanes

$\begin{array}{lll}\pi_{c} & - & \text { CPR } \\ \eta_{D} & - & \text { Diffuser efficiency }\end{array}$

$1-\eta_{D} \quad-\quad$ Diffuser loss coefficient

$\gamma \quad-\quad$ "Relative" diffuser loss coefficient

$\psi \quad-\quad$ Pressure coefficient: $\Delta p / \frac{\rho}{2} u_{2}^{2}$

$\varphi \quad-\quad \quad \quad$ Transport coefficient: $\dot{V} / \frac{\pi}{4} D_{2}^{2} u_{2}$ 


\section{Exposing the problem and steps of examination}

Nowadays, turbochargers, with maximum 50-60 mm impeller diameter, are more and more frequently designed with slightly BLIB. Backward leaning is not too significant, about $25-30^{\circ}$ to the radial direction (Tutak et al., 2015). At larger centrifugal compressors this variant is usually not used (Mayer, 1996; Zinner, 1985).

This paper would like to clear the questions of this solution. It examines how much the contradicted positive and negative effects compensate each other and what kind of advantages cause the BLIB has become competitive.

Centrifugal compressors with radial impeller blading practically can also be considered like the impeller blades would be leaned backward because the relative exit velocity vector leans backward due to the slip. In addition using BLIB the impeller exit relative velocity vector originally leans back following the direction of the trailing edge of the backward leaning blades.

Considering the blade stress the best solution is the radial blade impeller which accordingly allows the highest possible tangential speed. In this paper, due to its limitation, the stress analysis is out of our consideration, but it is evident that the backward and forward leaning impeller blades (the direction of leaning does not matter) suffer not only tensile but bending stress as well. Both the slip and the BLIB increase the tangential speed necessary for the same CPR. Of course with it the disc friction loss also increases, which is apparently disadvantage. While the slip is inherently arising from the work of impeller, the grade of blade backward leaning depends on the designer's decision, accordingly it must have some kind of advantages of this arrangement.

Due to the backward lean the channels between the blades have smaller diffuser angle (Ecker and Schnell, 1980; Daundel and Summer, 2000). This fact will be proven later. This is the reason, the efficiency of the impeller slightly increases, but it is partly annulated by the necessarily higher tangential speed. By the authors' examinations, the advantage of the BLIB can be found in the favorable change of compressor characteristics. Increasing the backward lean of the blades, the compressor stall margin moves towards reduced air mass flow rates. Due to this evidence the turbochargers even with small mass flow rate work without the stall, providing them higher flexibility. It will be proven later as well.

By the above-mentioned considerations the main steps of the paper:

The effect of the slip on the necessary tangential speed;

- Relationship between the exit angle of BLIB and the "virtual" slip and the determination of total slip;

- Determination of the relationship between the trailing edge angle of the impeller blades and the rate of divergence between impeller blades;

- Determination of the relationship between the decreasing divergence and the improving compressor efficiency;
- Examination, how the improving compressor efficiency and the increasing impeller tangential speed (or decreasing (PR) compensate each other;

- Higher compressor stall reserve (the stall margin moves to the direction of less air mass flow rate) due to the BLIB;

- Summary and evaluation of the results.

\section{The effect of the slip on the compressor work and its determination with thermal measurements}

The slip as an accompaniment of centrifugal compressor's operation is well known from Eckert and many others (Ecker and Schnell, 1980). At first a simple and well usable formula was created:

$$
\lambda=1-\left(\left(\pi \cos \beta_{2 s h}\right) / z\right)=c_{2 u \lambda} / u_{2}
$$

Definition of " $\beta_{2 s h}$ " can be seen in Fig. 1.

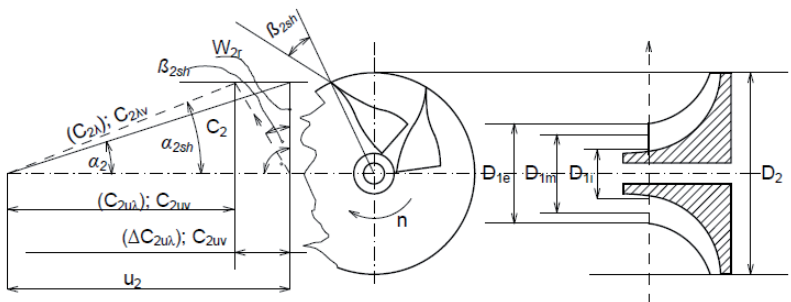

Fig. 1 Data of examined impeller and its exit velocity triangle without slip, $\beta_{2 s h}=25^{\circ} ; \mathrm{D}_{2}=56 \mathrm{~mm} ; \mathrm{D}_{1 \mathrm{e}}=41.5 \mathrm{~mm} ; \mathrm{D}_{1 \mathrm{~m}}=30.86 \mathrm{~mm} ; \mathrm{D}_{1 \mathrm{i}}=13.5 \mathrm{~mm} ; \mathrm{z}=12$

In some cases trying to achieve higher precision more complicated formulae are used, which includes the relative size of the compressor.

$$
\lambda=\left[1+\left\{\left(2 \pi \cos \beta_{2 s h}\right) / 3 z\left(1-\bar{D}_{1 m}\right)\right\}\right]^{-1}
$$

Where:

$$
\bar{D}_{1 m}=\left\{\left[0,5\left(D_{1 i}^{2}+D_{1 e}^{2}\right)\right]^{0.5}\right\} / D_{2}
$$

The slip factor is about $0.75-0.85$. Its value actually depends on the number of impeller blades and through it the inertia of intaking air. If $\beta_{2 s h}>0$, the rate of divergence between impeller blades decreases. Practically its effect is the same like the impeller would have more blades. Although, increasing the number of impeller blades means larger blade surface and higher friction loss. Splitter blades, that are located between each neighbouring "full" blade, are used in a vast number of applications, and provide a solution for the problem. For different size and type of centrifugal compressors, the optimum number of blades is determinable to achieve the best possible efficiency of the impeller. The compressor efficiency is independent on the value of " $\lambda$ ", because the ratio of input and output work of compressor remains approximately constant. With axial air intake (without inlet guide vanes) the Euler equation, considering $c_{2 u \lambda}=\lambda \cdot u_{2}$, is $w_{\text {ceff }}=\lambda \cdot u_{2}^{2}$.

The CPR " $\pi_{c}$ " versus tangential speed " $u_{2}$ " can be seen at various values of slip factor " $\lambda$ " in Fig. 2. 


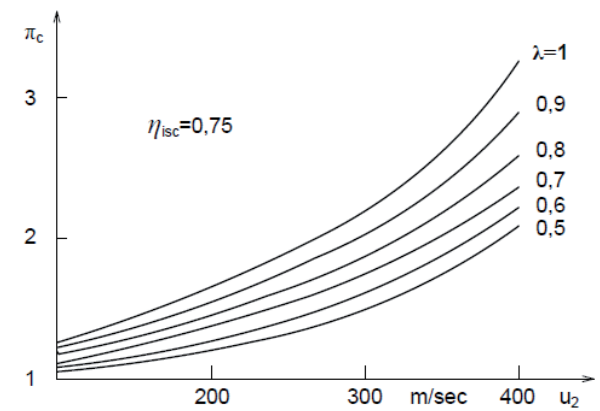

Fig. 2 CPR as a function of tangential speed assuming constant compressor isentropic efficiency and different slip factors

A significant change of " $\lambda$ " is no longer negligible. In Fig. 1 the "real" and "virtual" slip is illustrated through a velocity triangle and indicating, that their effect is the same. Strictly it is valid for the "virtual" slip so the symbol "real" slip factor is enclosed in parentheses.

" $\lambda$ ", the slip factor of radial bladed impeller, can be determined as follows:

Compressor real input work can be calculated by the Euler equation. By the equation, the real enthalpy raise is a function of the " $u_{2}$ " tangential speed irrespectively of compressor efficiency. The temperature difference between the compressor inlet and exit section can be measured and from that the real enthalpy rise can be calculated. From the energy equation, " $\lambda$ " can be determined.

$$
w_{\text {ceff }}=\lambda \cdot u_{2}^{2}=c_{\text {pair }}\left(T_{2 r e}-T_{0}\right)
$$

At BLIB the calculation of " $\lambda_{v}$ " is practically the same, except " $\lambda_{v}$ " "virtual" slip factor of the backward leaning, should be extracted from " $\lambda_{\Sigma}$ " "total" slip factor. The method was worked out earlier (known the estimated " $\lambda$ ") to determine the estimated RPM of high RPM impellers when its measuring was difficult.

\section{Energy properties of slightly BLIB}

As it is mentioned earlier, the backward leaning of the impeller blades slightly decreases the "real" slip, consequently the slip factor increases. This effect should be examined more precisely.

We start with the simplest formula (1). Let the number of impeller blades " $z$ " be 12 (symbols in Fig. 1).

If $\beta_{2 s h}=0^{\circ}$, then $\lambda=0.738$. If $\beta_{2 s h}=20^{\circ}$, then $\lambda=0.754$. The increment is $2-2.2 \%$. For the first estimation, it is of small importance.

Using the more precise formula (2) and the necessary data: $D_{1 e}=41.5 \mathrm{~mm} ; D_{1 i}=3.5 \mathrm{~mm} ; D_{2}=56 \mathrm{~mm}$. If $\beta_{2 s h}=0^{\circ}$, then $\lambda=0.8$. If $\beta_{2 s h}=20^{\circ}$, then $\lambda=0.8094$. The increase is $1-1.2 \%$, which is also negligible.

The increase of the slip factor is less important considering the slightly decreasing necessary tangential speed, but it is the indirect proof, that the backward leaning of impeller blades decreases the blade channel diffusion angle. As the duct narrows, which causes the virtual increase of the blade number (the real number of blades are unchanged), meanwhile the decreased blade duct diffusion angle slightly increases the impeller efficiency. The backward leaning of the impeller blades, without the slip, increase the tangential component of " $w_{2}$ " relative velocity vector, which direction just opposite to the " $u_{2}$ " tangential velocity vector. Thus, the effect of the BLIB is the same like the slip itself. In accordance with it between the " $\beta_{2 s h}$ " and the " $\lambda_{v}$ " virtual slip factor must be the definite relation. In the velocity triangle, can be seen in Fig. 1, the continuous line means " $\beta_{2 s h}=0^{\circ}$ ", while the broken line " $\beta_{2 s h}>0^{\circ}$ " situation (symbols in Fig. 1).

Starting from the definition of " $\lambda_{v}$ ":

$$
\begin{gathered}
\lambda_{v}=c_{2 u v} / u_{2}=\left(u_{2}-\Delta c_{2 u v}\right) / u_{2}=1-\Delta c_{2 u v} / u_{2} \\
\operatorname{tg} \alpha_{2 s h}=w_{2 r} / \lambda_{v} u_{2} ; \quad \operatorname{tg} \beta_{2 s h}=\Delta c_{2 u v} / w_{2 r} \\
\operatorname{tg} \beta_{2 s h}=\frac{\left(1-\lambda_{v}\right)}{\lambda_{v} \operatorname{tg} \alpha_{2 s h}} \\
\lambda_{v}=\frac{1}{\operatorname{tg} \beta_{2 s h} \operatorname{tg} \alpha_{2 s h}+1}
\end{gathered}
$$

In Fig. 3 the " $\lambda_{v}$ " versus " $\beta_{2 s h}$ " function can be seen at different " $\alpha$ " angles. (The difference between " $\alpha_{2 s h}$ " and " $\alpha_{2}$ " angles, for the first estimation, is negligible, about $2-3^{\circ}$, $\left.\operatorname{tg} \alpha_{2 s h}=\operatorname{tg} \alpha_{2} / \lambda_{v}\right)$.

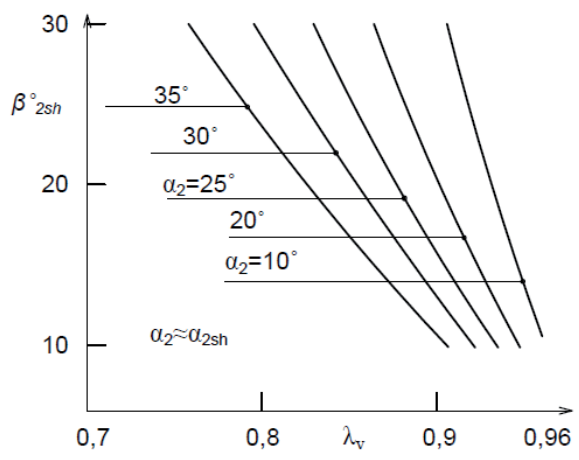

Fig. 3 The blade backward leaning angle " $\beta_{2 s h}$ " as a function of "virtual" slip " $\lambda_{v}$ " can be seen at different " $\alpha_{2}$ " angles

Noticeable, that even at small backward leaning the "virtual" slip factor " $\lambda$ " is equal with the "real" slip factor " $\lambda$ ", and in accordance with it, its effect is not negligible.

Notice: at any slip (virtual or real) the slip and the slip factor is opposite to each other, namely at increasing slip, the calculated slip factor decreases.

The total slip factor $\left(\lambda_{\Sigma}\right)$ is the result of "virtual" slip factor " $\lambda$ " "and the "real" slip factor " $\lambda$ " in accordance with the symbols of Fig. 1. In the velocity triangle (Fig. 1), the very small change of " $\lambda$ ", derived from the blade backward leaning, is not represented, but its existence has been proved earlier. Namely this change is so minor, that it is impossible to reveal in the drawing. 
Considering the definitions of " $\lambda$ " and " $\lambda$ ":

$$
\lambda_{\Sigma}=\frac{\left[u_{2}-\left(\Delta c_{2 u \lambda}+\Delta c_{2 u v}\right)\right]}{u_{2}}=\lambda+\lambda_{v}-1
$$

Assuming that the $\lambda=\lambda_{v}=0.8 \Rightarrow \lambda_{\Sigma}=0.6$.

\section{Determination of channel divergence rate between impeller blades and estimation of compressor efficiency improvement resulted by the BLIB}

The BLIB decreases the divergence rate of ducts, so the chance of compressor stall decreases and it can improve the compressor efficiency (Barton et al., 2006; Al-Hamdan and Ebaid, 2006). The examination was done on the large scale drawing imitating the real situation. The process can be seen in Fig. 4.

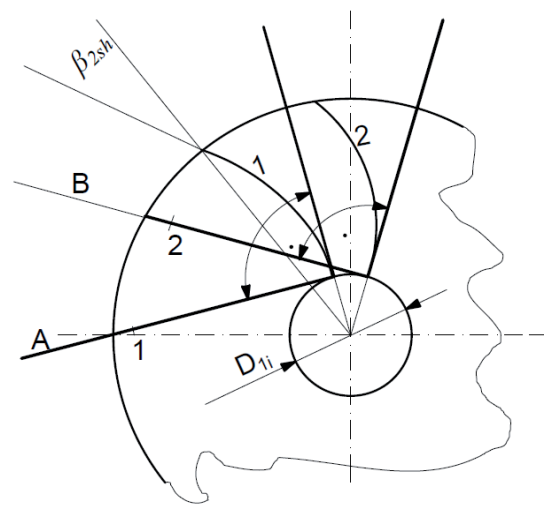

Fig. 4 Blades with different backward leaning and the determination of their divergence rate

The geometry and size of the impeller equivalent which can be seen in Fig. 1, so the model is based on a real impeller. The backward leaning blades were created from the circular arc. The blade roots are normal to the circle with diameter " $D_{1 i}$ ", consequently the center of the circles are located on " $\mathrm{A}$ " and "B" straight lines. The grade of backward leaning was varied by the change of circle radius. The examination range initiated from the radial blade impellers $\left(\beta_{2 s h}=0\right)$ to $\beta_{2 s h}=35^{\circ}$. Approximately, this $\beta_{2 s h}=35^{\circ}$ backward leaning means the final limit of moderate backward leaning of the impeller blades. The blades $(1 ; 2)$, illustrated by Fig. 4 represent $\beta_{2 s h}=27^{\circ}$ blade backward leaning. The change of the length of blade duct centerline, the diffuser angle and the blade trailing edge angle $\left(\beta_{2 \mathrm{sh}}\right)$ (Fig. 4), which actually describe the rate of backward leaning, was determined by the center points of circles drawn inside the duct.

The result is shown in Fig. 5.

The diffuser angle " $\alpha$ " with increasing " $\beta_{2 \text { sh }}$ " definitely decreases. Since the blade duct height during the examination remained unchanged accordingly it did not change the proportions. By the references (Eckert and Schnell, 1980;Bøhagen and Gravdahl, 2008; Mayer,1996; Tutak et al., 2015) the efficiency of the diffuser depends on two variables, namely the ratio of exit and inlet section of the diffuser (divergence ratio, Fig. 5)

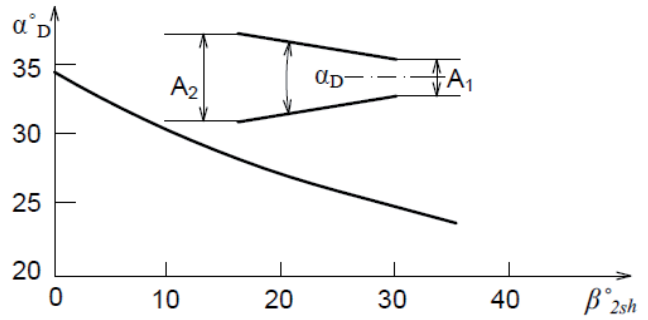

Fig. 5 Change of blade duct diffuser angle " $\alpha_{\mathrm{D}}$ " as a function of $\beta_{2 \mathrm{sh}}$ angle.

and the diffuser angle " $\alpha_{\mathrm{D}}$ ". By our examination, the diffuser divergence ratio $\left(A_{2} / A_{1}\right)$ ranged from 3.7 to 4.5 . As an average value, $A_{2} / A_{1}=4.1$ was accepted. Considering this value of $A_{2} / A_{1}$ the blade duct diffuser efficiency (used later for the approximation of impeller efficiency) as a function of the diffuser angle " $\alpha_{\mathrm{D}}$ " can be seen in Fig. 6.

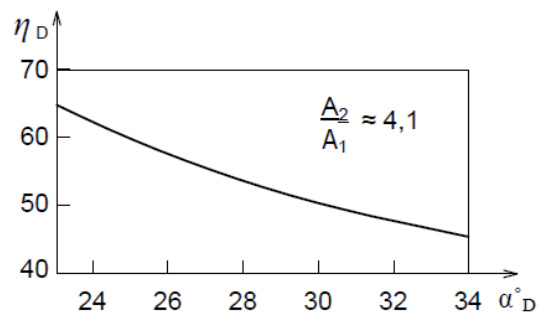

Fig. 6 The blade duct diffuser efficiency " $\eta_{D}$ " as a function of the diffuser angle " $\alpha_{D}$ ".

The possible improvement of impeller efficiency was approximated as follows:

For the backward leaning bladed compressor (impeller and diffuser) We gave $150 \mathrm{~K}$ as a real temperature rise (Fig. 7). The compressor's degree of reaction, considering the moderate backward leaning " $r_{c}=0.55$ ". The average isentropic efficiency " $\eta_{\text {isc }}$ " of the compressor is $0.73\left(\eta_{\text {is rot }}=0.72 ; \eta_{\text {is gu }}=0.74\right)$. Considering these data, the expected real turbocharging CPR is 3.1 .

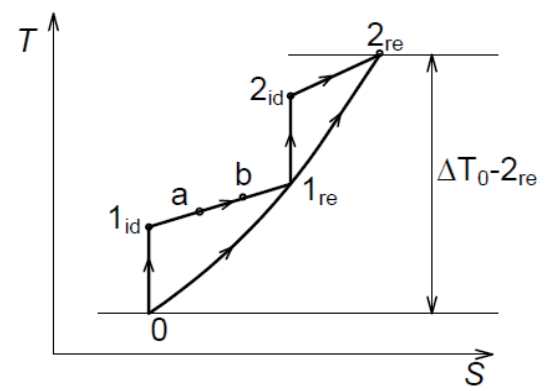

Fig. 7 The compressor processes (impeller and diffuser) in temperature versus entropy diagram.

The impeller loss expressing by the additional temperature rise " $\Delta T_{\text {lid-lre }}$ " is $23.1 \mathrm{~K}$. The approximated distribution of this loss (symbols in Fig. 7) as follows: incidence loss " $\Delta T_{\text {lid-a }}$ " is $14-16 \%(3.5 \mathrm{~K})$, disk friction loss " $\Delta T_{a-b}$ " is $39-41 \%(9.2$ $\mathrm{K})$, diffusion blading loss is " $\Delta \mathrm{T}_{\mathrm{b}-1 \mathrm{re}}$ " is $44-46 \%(10.4 \mathrm{~K})$ (El-Zahaby et al., 2012; Diez, Torregrosa, Pothas, 2011; 
Watson and Janota, 1982). The incidence loss and disk friction loss was assumed to be constant while diffusion blading loss $(10.4 \mathrm{~K})$ was connected to radial blade arrangement as an initial approximation. At increasing backward leaning $\left(\beta_{2 s h}>0\right)$ diffusion blading loss is decreased proportionally with the raise of diffuser efficiency " $\eta_{D}$ " (or fall of diffuser loss coefficient " $1-\eta_{D}$ ").

Considering $\left(\beta_{2 \mathrm{sh}}=0\right)$ as initial base we defined a new, so called (relative) diffuser loss coefficient (7):

$$
\gamma=\frac{1-\eta_{D \beta_{2 s h}=0}}{1-\eta_{D \beta_{2 s h}>0}}
$$

This coefficient is a function of " $\beta_{2 s h}$ ". Using the diagrams in Fig. 5 and 6, the value of " $\gamma$ " as a function of " $\beta_{2 s h}$ " changes as can be seen in Table 1 .

Table 1 " $\gamma$ " as a function of " $\beta_{2 s h}$ "

\begin{tabular}{ccccc}
\hline$\beta_{2 \text { sh }}$ & 35 & 25 & 10 & 0 \\
\hline$\alpha_{\mathrm{D}}$ & 23 & 25 & 30 & 34 \\
\hline$\eta_{\mathrm{D}}$ & 0.65 & 0.6 & 0.5 & 0.46 \\
\hline$\gamma$ & 0.648 & 0.741 & 0.926 & 1 \\
\hline
\end{tabular}

Using the definition of compressor efficiency " $\eta_{i s c}$ " (symbols in Fig. 8):

$$
\eta_{i s c}=\frac{\left(\Delta T_{0-2 r e}-\Delta T_{1 r e-2 r e}-\Delta T_{1 i d-a}-\Delta T_{a-b}-\Delta T_{b-1 r e} \cdot \gamma\right)}{\Delta T_{0-2 r e}}
$$

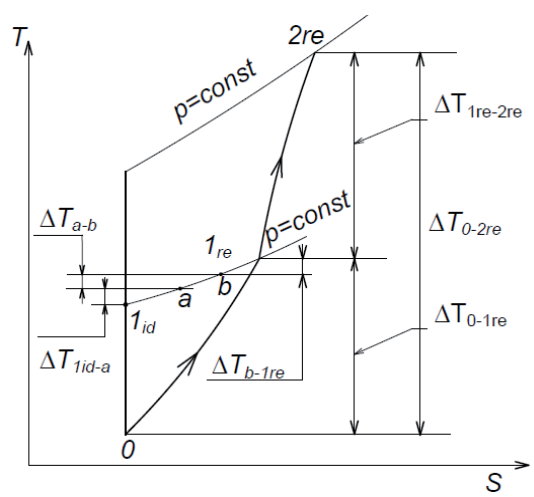

Fig. 8 The symbols of the compressor processes (impeller and diffuser) to determine the efficiency changes.

The change of compressor isentropic efficiency can be seen in Fig. 9. Until the practically used backward lean limit $\left(\beta_{2 \text { shmax }}\right.$ $\sim 30-35^{\circ}$ ) the efficiency improvement is maximum $2.5 \%$. In our opinion this improvement of compressor efficiency, despite the disadvantages, justifies the using of BLIB.

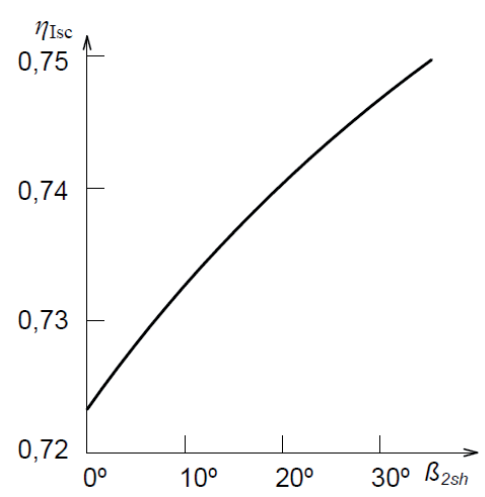

Fig. 9 The compressor isentropic efficiency " $\eta_{i s c}$ " as a function of the " $\beta_{2 s h}$ ".

Of course, using other less real initial approximations the results can give other, but not significantly different results, but it is quite clear, that the backward leaning of impeller blades has tendency to improve the compressor isentropic efficiency " $\eta_{i s c}$ ".

Another important question, considering the usefulness of BLIB requires further examination, namely whether its positive effect on isentropic efficiency is able to outnumber the decrease of the CPR at same tangential speed. The examination was done on the basis of the diagram Fig. 3. As can be seen there, between the " $\beta_{2 s h}$ " and the " $\lambda_{v}$ " virtual slip factor must be the definite relation at given " $\alpha_{2 s h}$ " angle. The isentropic efficiency, while $\beta_{2 s h}=0^{\circ}$, is 0.723 (Fig. 9). Assuming it as an initial value and using the $\beta_{2 s h}=f(\lambda)$ function, the change of CPR at increasing impeller backward leaning, keeping the tangential speed constant, can be determined. Since " $\beta$,s" depends on " $\alpha_{2 s h}$ ", the chosen " $\alpha_{2 s h}$ " for the examination is $25^{\circ}$. In accordance with the Euler equation the relationship among the " $\pi_{c}$ ", " $\beta_{2 s h}$ " and " $\lambda_{\Sigma}$ " is expressed by the Eq. (9).

$$
\pi_{c}=\left[\left\{\left(\lambda_{\Sigma} u^{2} \eta_{i s c}\right) /\left(c_{p \text { air }} T_{0}\right)\right\}+1\right]^{\kappa /(\kappa-1)}
$$

Where " $\lambda_{\Sigma}$ " was defined by Eq. (6).

Values of isentropic efficiency correspond to the diagram in Fig. 9. For the higher precision of examination Eq. (1) is used to define " $\lambda$ ". If $\beta_{2 s h}=0^{\circ}$ and $z=12$, then $\lambda_{v}=0.738$, while if $\beta_{2 s h}=35^{\circ}$, then $\lambda_{v}=0.786$. The results can be seen in Fig. 10:

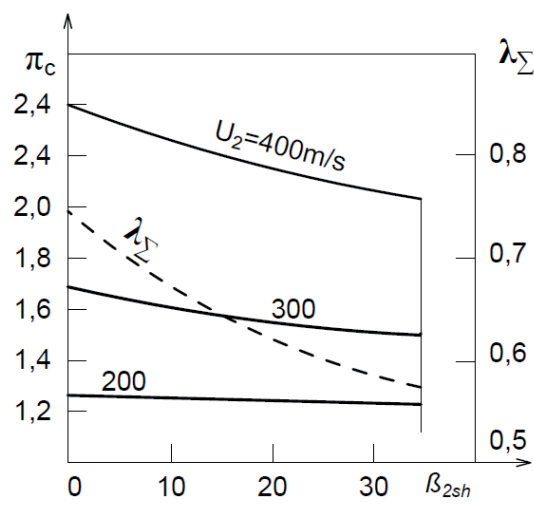

Fig. 10 The change of " $\pi_{c}$ ", and " $\lambda_{\Sigma}$ " as a function of " $\beta_{2 s h}$ " at two different tangential speed. 
By my examinations, at increasing " $\beta_{2 s h}$ ", the decrease of " $\lambda_{\Sigma}$ " is not compensated totally by the higher " $\eta_{i s c}$ ". The value of " $\pi_{c}$ ", considering normal tangential speed range, at the best case is constant, but expectedly not significantly, but decreases. It was mentioned earlier, that the backward leaning impellers surely must have other advantage, which made it reasonable to use them in centrifugal compressors of small size turbochargers. This fundamental advantage can be found in the favorable change of compressor characteristics. The operational point of turbochargers, especially the small, vehicle turbochargers, depends dramatically on the rate of power (related to traffic, road and load conditions), i.e. the flexibility of the turbochargers is especially important. This ability of compressors is enhanced significantly by backward leaning impellers. Analyzing this favorable transformation of compressor characteristics, it is immediately clear why the BLIB are not used in larger (rail transport and ship engines) turbochargers. The operational point of turbochargers, in rail transport, but especially in ship engines practically fixed in the compressor characteristics. In accordance with it, smaller loads (small air consumption) are not definitely necessary the stabile operation of the compressor.

In aircraft engines, because of the very high rotational speed, it is not practical to increase the originally large tensile stress with an additional bending stress, caused by BLIB. In aircraft propulsion, the stabile operation is provided with other methods (Beneda, 2013).

We start the examination with a radial bladed centrifugal compressor characteristics at a given RPM whose pressure coefficient " $\psi$ " as a function of transport coefficient " $\varphi$ " can be seen in Fig. 11.

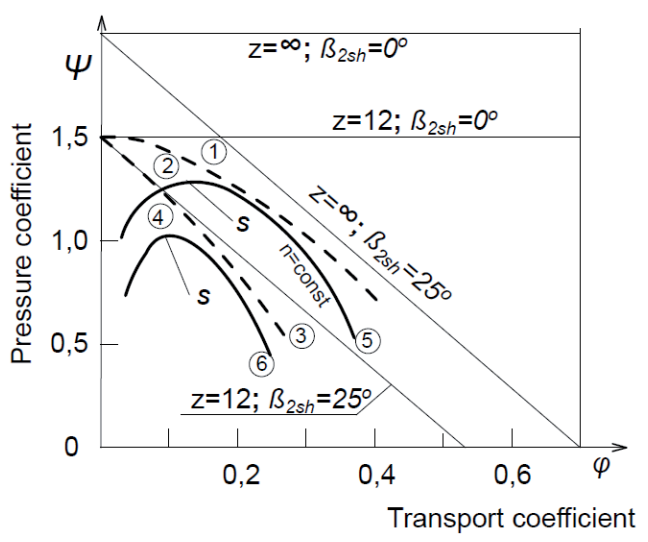

Fig. 11 The change of pressure coefficient " $\psi$ " as a function of transport coefficient " $\varphi$ " of the examined compressor.

Symbols in Fig. 11: 1;3: disk friction loss; 2;4: incidence loss; 5: calculated characteristics of " $\beta_{2 s h}=0$ "; 6 : calculated characteristics of " $\beta_{2 s h}=25$ "; S: stability margin. Determining the characteristics of this type and sized compressor, real loss coefficients were taken into consideration (Diez, Torregrosa,
Pothas, 2011; Watson and Janota, 1982). The procedure was done by the references Ecker, Schnell (1980), (Daundel and Summer, 2000). The points symbolized by " $S$ " represent the stability margin in Fig. 11. ". If $\beta_{2 s h}=0^{\circ}$, then $\varphi=0.15$, while if $\beta_{2 s h}=25^{\circ}$, then $\varphi=0.1$. Using moderate backward leaning impellers, it is clearly visible, that the stability margin moves to the lower " $\varphi$ " values, i.e. providing stable work of compressor even at the lower mass flow rate.

The pressure coefficient " $\psi$ " of backward leaning impellers decreases. It was predictable and was proven earlier with the examination of CPR assuming unchanged tangential speed. The improvement of stability reserve mainly originated from the lower divergence rate $\left(\beta_{2 s h}>0\right)$, consequently tends less for flow separation and stall. Conclusively, using the backward leaning impeller can be one of the methods to avoid the centrifugal compressor stall (surge) (Zhao et al., 2012; Beneda, 2013).

Important to underline, that this advantage is true only for moderate backward leaning. If $\beta_{2 s h}>30-35^{\circ}$, " $\pi_{c}$ " significantly decreases despite the increasing " $\eta_{\text {isc }}$ ". This disadvantage (higher RPM, higher stress, larger size) is the main reason, that there are no larger impellers with backward leaning blades.

\section{Conclusion}

Over the last decade, compressors with moderate BLIB have appeared in turbochargers.

The BLIB decrease the divergence rate of blade ducts, and this can improve the compressor efficiency by $2-2.5 \%$.

The slip factor " $\lambda$ ", due to higher " $\beta_{2 \mathrm{sh}}$ " increases by some percentage.

The backward leaning, considering the same CPR increases the necessary tangential speed.

Impeller with backward leaning blades virtually does like an impeller with lower slip factor and it is called "virtual" slip factor " $\lambda_{\mathrm{v}}$ ".

Between the " $\beta_{2 \text { sh }}$ " angle of backward leaning and the " $\lambda_{\mathrm{v}}$ " virtual slip factor, there is a definite relation at different " $\alpha_{2 \mathrm{sh}}$ " angles.

The total slip factor $\left(\lambda_{\Sigma}\right)$ is the result of "virtual" slip factor " $\lambda_{\mathrm{v}}$ " and the "real" slip factor " $\lambda$ ": $\lambda_{\Sigma}=\lambda+\lambda_{v}-1$

The improved compressor efficiency cannot counteract the negative effect of " $\lambda_{\Sigma}$ ", consequently at the same tangential speed with increasing " $\beta_{2 \text { sh }}$ ", CPR (not significantly) decreases about $6-10 \%$.

The advantage of backward leaning impellers is that the stability margin moves to the direction of the smaller mass flow rate of air. The rate of this change, considering " $\varphi$ " transport factor $\Delta \varphi=0.2-0.25 \varphi$. In accordance with it, that kind of centrifugal compressors is adapted more flexible way to the significantly changing operational situations. 


\section{References}

Al-Hamdan, Q. Z., Ebaid, M. S. Y. (2006) Modelling and Simulation of a Gas Turbine Engine for Power Generation. ASME Journal of Engineering for Gas Turbines and Power. 128 (2). pp. 302-311. DOI: 10.1115/1.2061287

Barton, M. T., Mansour, M. L., Liu, J. S., Palmer, D. L. (2006) Numerical Optimization of a Vaned Shroud Design for Increased Operability Margin in Modern Centrifugal Compressors. ASME Journal of Turbomachinery 128 (4). pp. 627-631. DOI: 10.1115/1.2187526

Beneda, K. (2013) CFD Simulation of Blade Load Distribution Control as Active Centrifugal Compressor Surge Suppression. Acta Avionica 15 (25). pp. 13-20.

Beneda, K. (2013) LQ Optimal Control of Variable Inducer Shroud Bleed for Centrifugal Compressor Surge Suppression. In: Third Scientific Workshop of Faculty Doctoral Schools. Budapest, May 28, 2013.

Bøhagen, B., Gravdahl, J. T. (2008) Active Surge Control of Compression System Using Drive Torque. Automatica. 44 (4). pp. 1135-1140. DOI: $10.1016 /$ j.automatica.2007.11.002

Daundel, H., Sumser, S. (2000) Turbochargers. (Abgasturboauflading.) Daimler Chrysler A G., Stuttgart. (in German)

Diez, F. J., Torregrosa, M. M., Pothos, S. (2011) A Comparison between Round Turbulent Jets and Particle-laden Jets in Cross-flow by Using Timeresolved Stereoscopic Particle Impage Velocimetry. ASME Journal of Fluids Engineering. 133 (9). DOI: 10.1115/1.4004815
Eckert, B., Schnell, E. (1980) Axial und Radialkompressoren: Anwendung, Theorie, Berechnung. (Axial and Centrifugal Compressors: Application, Theory, Calculation.), Springer, Berlin. (in German)

El-Zahaby, A., El-Shenawy, E., Ziada, Z., Eldin, K., Salem, M. (2012) Modelling of Compression System with Recirculation Technique and Validation Using PIV. In: American Control Conference (ACC), 2012 . pp. 392397, 27-29 June 2012. DOI: 10.1109/ACC.2012.6314877

Mayer, M. (1996) Effective use of exhaust gas energy. Verlag moderne Industrie, Landsberg/Lech.

Tutak, W. Lukács, K., Szwaja, S., Bereczky, Á. (2015) Alcohol-diesel fuel combustion in the compression ignition engine. Fuel. 154 . pp. 196-206. DOI: $10.1016 /$ j.fuel.2015.03.071

Watson, M. Janota, M. S. (1982) Turbocharging the Internal Combustion Engine. Macmillam, London.

Zhao, D., Blunier, B., Dou, M., Miraou, A. (2012) Control of an Ultra High Speed Centrifugal Compressor for the Air Management of Fuel Cell Systems. In Industry Applications Society Annual Meeting (IAS), 2012 IEEE. pp.1-8, 7-11 Oct. 2012. DOI: 10.1109/IAS.2012.6374007

Zinner, K. (1985) Aufladung von Verbrennungsmotoren. (Charging of combustion engines.), Springer Verlag. (in German) 